\title{
Prognostic Value of Lymph Node Characteristics in Patients with Cervical Cancer Treated with Radical Hysterectomy
}

\author{
Yoon Hee Lee ${ }^{1-3}$ \\ Gun Oh Chong (D) ${ }^{1-3}$ \\ $\mathrm{Su}$ Jeong $\mathrm{Kim}^{1,2}$ \\ Ja Hyun Hwang ${ }^{1,2}$ \\ Jong Mi Kim (iD ${ }^{1,2}$ \\ Nora Jee-Young Park ${ }^{3,4}$ \\ Dae Gy Hong ${ }^{1,2}$ \\ 'Department of Obstetrics and \\ Gynecology, School of Medicine, \\ Kyungpook National University, Daegu, \\ Republic of Korea; ${ }^{2}$ Department of \\ Obstetrics and Gynecology, Kyungpook \\ National University Chilgok Hospital, \\ Daegu, Republic of Korea; ${ }^{3}$ Clinical \\ Omics Research Center, School of \\ Medicine, Kyungpook National \\ University, Daegu, Republic of Korea; \\ ${ }^{4}$ Department of Pathology, School of \\ Medicine, Kyungpook National \\ University, Daegu, Republic of Korea
}

Correspondence: Gun Oh Chong Kyungpook National University Chilgok Hospital, 807 Hogukno, Buk-Gu, Daegu, 700-720, Republic of Korea

Tel +82-53-200-2684

Fax +82-53-200-2028

Email gochong@knu.ac.kr
Objective: This study evaluated the prognostic value of various lymph node (LN) characteristics, including the lymph node ratio (LNR), in patients with cervical cancer treated with radical hysterectomy.

Methods: In this retrospective study, 260 patients with cervical cancer who had undergone radical hysterectomy with pelvic or paraaortic lymphadenectomies were included. LN characteristics related to several LN statuses included total LN counts, LN metastasis, total positive LN counts, LNR, and levels of lymphadenectomy. LNR was defined as the number of metastatic LNs divided by the total number of LNs harvested. Univariate and multivariate analyses for disease-free survival (DFS) and overall survival (OS) were performed using the clinicopathological and LN characteristics.

Results: Based on receiver-operating characteristics curve analysis, the cut-off value of LNR was 0.0625 . Multivariate analysis revealed that high LNR was significantly related to tumor recurrence (hazard ratio [HR], 5.182; 95\% confidence interval [CI], 2.424-11.075; $p<$ 0.0001). After adjusting for clinicopathological factors, LNR was also independent prognostic factor for predicting tumor recurrence (HR, 5.930; 95\% CI, 2.114-16.634; $p=$ 0.0007). However, total retrieved LN counts and level of lymphadenectomy were not associated with survival outcomes.

Conclusion: LNR may be a prognostic biomarker for predicting disease recurrence in cervical cancer treated with radical hysterectomy.

Keywords: cervical cancer, lymph node ratio, radical hysterectomy, prognosis

\section{Introduction}

Radical hysterectomy is the standard treatment for early-stage cervical cancer, and adjuvant chemoradiotherapy should be considered in patients with high-risk factors, including lymph node (LN) metastasis, positive resection margin, and parametrial invasion. ${ }^{1,2} \mathrm{LN}$ involvement is a strong prognostic parameter for patients with cervical cancer receiving radical surgery. ${ }^{3}$ Moreover, lymphatic dissemination to the regional $\mathrm{LN}$ has been suggested as the main route of metastasis in cervical cancer. ${ }^{4}$ So, regional LN dissection is necessary to decide the adjuvant treatment and survival outcomes.

Several factors related to the LN status affect prognosis in early-stage cervical cancer, including the total number of LN retrieved, ${ }^{5,6}$ negative LN counts, ${ }^{7}$ the number of positive $\mathrm{LNs}^{8},{ }^{8}$ and localization of metastasis. ${ }^{8}$ Recently, several studies have demonstrated that the lymph node ratio (LNR) had been suggested as a newly 
emerging prognostic factor in cervical cancer. ${ }^{9-11}$ LNR is defined as the ratio between the number of positive LNs and removed LNs, and ratio-based nodal assessment may be a relatively more objective measure of nodal tumor burden compared with the number-based nodal category. Till now, previous majoritic studies have demonstrated the prognostic value of each factor related to $\mathrm{LN}$ status. Moreover, the prognostic value of these several factors related to $\mathrm{LN}$ status was controversial.

Lymphadenectomy has been discussed concerning prognostic, predictive, and therapeutic aspects in gynecological cancers. ${ }^{12}$ The therapeutic approach removes all nodes and reduces the risk of recurrence. Theoretically, a higher number of acquired LN counts could accurately assess LN status, reducing occult LN metastasis risk. Previous studies showed that more extensive lymphadenectomy improved survival outcomes in cervical cancer. ${ }^{5,13}$ Previously, we introduced an extended lymphadenectomy, including the superior and inferior gluteal, presacral, common iliac, and lower paraaortic nodes as part of radical hysterectomy for cervical cancer. ${ }^{14}$ Using this approach, we could harvest more LNs and have a high rate of metastatic nodes; however, long-term survival benefit was not verified.

The Laparoscopic Approach to Cervical Cancer (LACC) trial, a randomized, open-label, noninferiority study that compared minimally invasive radical hysterectomy to open radical hysterectomy, found that minimally invasive surgery was associated with a higher risk of recurrence and death compared with open surgery. ${ }^{15}$ Therefore, the difference between the surgical approaches during lymphadenectomy may influence the survival outcomes of patients with cervical cancer.

This study evaluated the prognostic value of several factors related to LN status, such as total LN counts, total positive LN counts, and LNR. Moreover, we evaluated the prognostic value of several factors related to $\mathrm{LN}$ status according to LN metastasis. Furthermore, long-term survival benefits of lower paraaortic lymphadenectomies or extended lymphadenectomy were evaluated compared with conventional pelvic lymphadenectomy.

\section{Materials and Methods}

\section{Patients}

In this study, 260 patients diagnosed with biopsy-proven cervical cancer were enrolled between November 2007 and December 2016. Retrospective data collection and analysis were approved by the Institutional Review
Board of Kyungpook National University Chilgok Hospital (KNUCH 2020-03-011). The need for informed consent was waived due to the retrospective design of the study. This study was conducted in accordance with the Declaration of Helsinki and all patient data was kept confidential. The patients were clinically staged according to the 2009 International Federation of Gynecologic Obstetrics (FIGO) staging system. ${ }^{16}$ Only patients with the completion of 3 years of follow-up and $\geq 10$ retrieved total LN counts were included. The protocol of cancer staging included a pelvic examination under general anesthesia, conization, magnetic resonance imaging of the pelvis, and positron emission tomography/computed tomography. Clinical and pathological parameters were reviewed and retrieved, including age, FIGO stage, histology, primary tumor size, lymphovascular invasion (LVI), deep stromal invasion, parametrial invasion, resection margin status, and LN characteristics.

\section{Surgical Procedures and Adjuvant Treatments}

Gynecologic oncologists performed all operations. All patients underwent type $\mathrm{C} 1$ radical hysterectomy classified by Querleu and Morrow. ${ }^{17}$ Conventional pelvic lymphadenectomy was performed in a standard fashion, and LNs in external and internal iliac and obturator nodal stations were removed. Low paraaortic lymphadenectomy added presacral, low paraaortic, and common iliac LNs to conventional pelvic lymphadenectomy. Extended lymphadenectomy was defined addition of superior and inferior gluteal LNs with low paraaortic lymphadenectomy. Paraaortic lymphadenectomy was performed if pelvic LN involvement was confirmed by frozen section or preoperative imaging studies. Adjuvant concurrent chemoradiotherapy was conducted for positive LNs, parametrial invasion or positive surgical margin, adjuvant radiotherapy, or chemotherapy for intermediate-risk factors.

\section{LN Characteristics}

LN characteristics related to several LN statuses included total LN counts, LN metastasis, total positive LN counts, LNR, and levels of lymphadenectomy. LNR was defined as the number of metastatic LNs divided by the total number of LNs harvested. A receiver-operating characteristic (ROC) curve analysis was performed to identify an optimal cut-off of each factor related to LN status to predict recurrence and death. 


\section{Clinical Follow-Up}

Clinical follow-up of patients was performed every 3 months for 2 years, then every 6 months after 2 and up to 5 years, and annually after that. Failure was defined as biopsy-proven recurrence or documentation of disease progression on serial imaging studies.

\section{Statistical Analysis}

Continuous data were expressed as mean \pm standard deviation, and categorical data were presented as frequency and percentage. The time to event was calculated as the time interval from the date of diagnosis to the date of the first clinical or imaging findings that suggested disease recurrence. Student's $t$-test evaluated the differences between subsets, and differences between proportions were compared with the chi-square test or Fisher's exact test. Survival curves of prognostic factors were estimated using the Kaplan-Meier method, and differences between subgroups were compared using the Log rank test. A univariate Cox proportional hazards model was used to determine hazard ratios of prognostic factors for disease-free survival (DFS) and overall survival (OS). A forward, stepwise multivariate Cox proportional hazards model was used to assess the potential independent effects of prognostic factors for DFS and OS. An estimated hazard ratio (HR) with a $95 \%$ confidence interval $(95 \%$ CI) was calculated.

The MedCalc statistical package (v.12.3.0.0, MedCalc Software, Mariakerke, Belgium) was used for statistical analysis. A $P$ value of less than 0.05 was considered statistically significant.

\section{Results}

\section{Clinicopathologic and LN Characteristics}

The clinicopathological and LN characteristics of the study participants are listed in Table 1. The predominant FIGO stage was IB1 $(\mathrm{n}=187$ [71.9\%]), followed by IB2 $(\mathrm{n}=34$ [13.1\%]), IIB $(n=21[8.1 \%])$, IIA1 $(n=12$ [4.6\%]) and IIA2 $(\mathrm{n}=6[2.3 \%])$. The histological types of cervical cancer were as follows: squamous cell carcinoma $(\mathrm{n}=178$ [68.5\%]) and adenocarcinoma/adenosquamous carcinoma $(\mathrm{n}=82$ [31.5\%]). Minimally invasive surgery was performed in 233 patients $(89.6 \%)$, whereas open surgery was performed in 27 patients $(10.4 \%)$.

The mean number of total acquired LNs was $37.7 \pm$ 18.2 and total positive $\mathrm{LN}$ counts was $0.55 \pm 1.97$. The mean LNR was $0.014 \pm 0.048$. LN metastasis was found in
49 patients $(18.8 \%)$. Conventional pelvic lymphadenectomy was performed in 106 patients (40.8\%), low paraaortic lymphadenectomy in 52 patients $(20.0 \%)$, and extended lymphadenectomy in 102 patients $(39.2 \%$, Table 1$)$.

\section{Treatment Outcomes}

Fifty patients received concurrent adjuvant chemoradiotherapy for high-risk factors, including positive LNs, parametrial invasion, or positive surgical margin, and 30 patients received adjuvant radiotherapy for intermediaterisk factors. Forty-seven patients received adjuvant chemotherapy for high- or intermediate-risk factors.

After a median follow-up of 58 months (6-132 months), 35 patients $(13.5 \%)$ had a recurrence, and 14 patients $(5.4 \%)$ had died due to disease progression.

\section{Survival Analysis in All Patients}

Multivariate analysis with the forward stepwise Cox proportional hazards model demonstrated that only LNR (HR, 5.182; 95\% confidence interval [CI], 2.424$11.075 ; p<0.0001)$ remained as significant prognostic factors for DFS (Table 2). FIGO stage (HR, 3.349; 95\% CI, 1.168-9.608; $p=0.0246)$ and histology (HR, 3.041; $95 \%$ CI, $1.050-8.807 ; p=0.0404$ ) for OS (Table 3). The Kaplan-Meier survival plots revealed significant differences in DFS and OS when stratified by LNR (Figure 1A and B).

\section{Survival Analysis in Patients with LN Metastasis}

Only LNR (HR, 7.795; 95\% CI, 1.679-36.182; $p=$ 0.0087) was an independent biomarker for predicting tumor recurrence (Supplementary Table 1); however, there was no independent biomarker for predicting death (Supplementary Table 2) in multivariate analysis.

\section{Survival Analysis in Patients without LN Metastasis}

Multivariate analysis showed positive margin (HR, 3.544; 95\% CI, 1.391-9.030; $p=0.0080$ ) was a significant prognostic factor for DFS (Supplementary Table 3) and histology (HR, $4.117 ; 95 \%$ CI, $1.029-16.477 ; p=0.0455)$ for OS (Supplementary Table 4). However, LN characteristics were not associated with prognosis in patients without $\mathrm{LN}$ metastasis. 
Table I Clinicopathologic and Lymph Node Characteristics of Cervical Patients with and without Recurrence

\begin{tabular}{|c|c|c|c|c|}
\hline Variables & All $(n=260)$ & No Recurrence $(n=225)$ & Recurrence $(n=35)$ & $P$ value \\
\hline Age (years) & $48.5 \pm 9.9$ & $49.1 \pm 9.8$ & $43.0 \pm 10.7$ & 0.1848 \\
\hline \multicolumn{5}{|l|}{ FIGO stage $(n, \%)$} \\
\hline$|B|$ & I87 (7I.9) & $167(74.2)$ & $20(57.1)$ & 0.0273 \\
\hline IB2 & $34(13.1)$ & $25(\mathrm{II} . \mathrm{I})$ & $9(5.7)$ & \\
\hline IIAI & $12(4.6)$ & $9(4.0)$ & $3(8.6)$ & \\
\hline $\mathrm{IIA} 2$ & $6(2.3)$ & $4(1.8)$ & $2(5.7)$ & \\
\hline $\mathrm{IIB}$ & $21(8.1)$ & $20(8.9)$ & I (2.9) & \\
\hline \multicolumn{5}{|l|}{ Histology (n, \%) } \\
\hline SCC & $178(68.5)$ & $158(70.2)$ & $20(57.1)$ & 0.1211 \\
\hline ACIASC & $82(31.5)$ & $67(29.8)$ & $15(42.9)$ & \\
\hline Tumor size $(\mathrm{cm})$ & $2.2 \pm 1.8$ & $2.1 \pm 1.8$ & $2.9 \pm 1.6$ & 0.0136 \\
\hline LVI (n, \%) & $89(34.2)$ & $77(34.2)$ & $12(34.3)$ & 0.9941 \\
\hline Deep stromal invasion (n, \%) & $116(44.6)$ & $94(4 I .8)$ & $22(62.9)$ & 0.0198 \\
\hline Parametrial invasion (n, \%) & $37(14.2)$ & $30(13.3)$ & $7(20.0)$ & 0.2946 \\
\hline Positive vaginal margin ( $\mathrm{n}, \%)$ & $41(15.8)$ & $31(13.8)$ & $10(28.6)$ & 0.0258 \\
\hline \multicolumn{5}{|l|}{ Type of Surgery } \\
\hline MIS & $233(89.6)$ & $202(89.8)$ & $31(88.6)$ & 0.8280 \\
\hline Open & $27(10.4)$ & $23(10.2)$ & $4(11.4)$ & \\
\hline \multicolumn{5}{|l|}{ LN Characteristics } \\
\hline Total counts $(n)$ & $37.7 \pm 18.2$ & $37.9 \pm 18.5$ & $36.8 \pm 15.9$ & 0.7357 \\
\hline $\mathrm{LN}$ metastasis $(\mathrm{n}, \%)$ & $49(18.8)$ & $38(16.9)$ & II (3I.4) & 0.0411 \\
\hline Total positive LN counts $(n)$ & $0.55 \pm 1.97$ & $0.43 \pm 1.58$ & $1.29 \pm 3.5 \mid$ & 0.0165 \\
\hline LNR & $0.014 \pm 0.048$ & $0.011 \pm 0.045$ & $0.030 \pm 0.065$ & 0.0316 \\
\hline \multicolumn{5}{|l|}{ Level of lymphadenectomy (n, \%) } \\
\hline Pelvis & $106(40.8)$ & $96(42.7)$ & $10(28.6)$ & 0.1840 \\
\hline Pelvis + low paraaortic & $52(20.0)$ & $43(19.1)$ & $9(25.7)$ & \\
\hline Pelvis + extended & $102(39.2)$ & $86(38.2)$ & $16(45.7)$ & \\
\hline
\end{tabular}

Abbreviations: AC, adenocarcinoma; ASC, adenosquamous carcinoma; Cl, confidence interval; FIGO, International Federation of Gynecology and Obstetrics; HR, hazard ratio; LN, lymph node; LNR, lymph node ratio; MIS, minimally invasive surgery; SCC, squamous cell carcinoma.

Multivariate Analysis of LN

\section{Characteristics for DFS and OS After} Adjusting for Clinicopathologic Factors

The multivariate analysis performed for DFS and OS following the adjustment for the effects of clinicopathologic variables, which were statistically significant in the univariate survival analyses, revealed that only LNR was independent prognostic factors for DFS (HR, 5.930; 95\% CI, 2.114-16.634; $p=0.0007$; Table 4).

\section{Discussion}

This study evaluated the prognostic value of various $\mathrm{LN}$ characteristics in cervical cancer patients treated with radical hysterectomy. LNR $(>0.0625)$ was the most robust biomarker for predicting tumor recurrence among the various LN characteristics.

LNR combines information on the number of positive LNs and the total number of retrieved LNs. So, LNR has the advantage of reflecting the number of metastatic LNs and the extent of LN dissection ${ }^{18}$ and may better stratify patients regarding prognosis. The advantages of LNR over other parameters as follows: underestimation due to less aggressive dissection can be avoided, ${ }^{18}$ the thoroughness of the surgical dissection and pathological examination in reflected, and it is easy to calculate. ${ }^{9}$ The cut-off values for high LNR in cervical cancer differed for each previous study, ranging from 5\% to $40 \%{ }^{9}{ }^{9,10}$ LNR may appear relatively high in limited LN counts and may not reflect the exact tumor burden. In 
Table 2 Univariate and Multivariate Analyses of Clinical Variables and Lymph Node Characteristics for Prediction of Tumor Recurrence

\begin{tabular}{|c|c|c|c|c|c|c|}
\hline \multirow[t]{2}{*}{ Variables } & \multicolumn{3}{|c|}{ Univariate Analysis } & \multicolumn{3}{|c|}{ Multivariate Analysis } \\
\hline & HR & $95 \% \mathrm{Cl}$ & $\boldsymbol{P}$ & HR & $95 \% \mathrm{Cl}$ & $P$ \\
\hline $\begin{array}{l}\text { Age (years) } \\
\leq 40 \text { vs }>40\end{array}$ & 2.695 & I.079-6.732 & 0.0338 & & & \\
\hline $\begin{array}{l}\text { Stage } \\
\geq \mid \mathrm{IB} 2 \text { vs }|\mathrm{B}|\end{array}$ & 2.550 & I. $187-5.480$ & 0.0164 & & & \\
\hline $\begin{array}{l}\text { Histology } \\
\text { AC/ASC vs SCC }\end{array}$ & 1.729 & $0.844-3.54 I$ & 0.1347 & & & \\
\hline $\begin{array}{l}\text { Tumor size } \\
>2 \mathrm{~cm} \text { vs } \leq 2 \mathrm{~cm}\end{array}$ & 1.958 & $1.008-3.806$ & 0.0475 & & & \\
\hline Lymphovascular invasion & 1.014 & $0.503-2.044$ & 0.9695 & & & \\
\hline Deep stromal invasion & 2.291 & I. $17 \mid-4.485$ & 0.0155 & & & \\
\hline Parametrial invasion & 1.947 & $0.717-5.287$ & 0.1913 & & & \\
\hline Positive margin & 3.508 & $1.335-9.215$ & 0.0109 & & & \\
\hline $\begin{array}{l}\text { Type of surgery } \\
\text { MIS vs open }\end{array}$ & 0.734 & $0.229-2.357$ & 0.6034 & & & \\
\hline $\begin{array}{l}\text { Total LN counts } \\
\leq 30 \text { vs }>30 \\
\text { LN metastasis }\end{array}$ & $\begin{array}{l}1.547 \\
2.657\end{array}$ & $\begin{array}{l}0.783-3.057 \\
1.107-6.378\end{array}$ & $\begin{array}{l}0.2092 \\
0.0287\end{array}$ & & & \\
\hline $\begin{array}{l}\text { Total positive LN counts } \\
>\text { I vs } \leq \text { I }\end{array}$ & 5.423 & $1.702-17.277$ & 0.0042 & & & \\
\hline $\begin{array}{l}\text { LNR } \\
>0.0625 \text { vs } \leq 0.0625\end{array}$ & 25.577 & $7.015-108.408$ & $<0.0001$ & 5.182 & $2.424-11.075$ & $<0.000$ I \\
\hline $\begin{array}{l}\text { Level of LN dissection } \\
\text { Pelvis vs + low paraaortic } \\
\text { Pelvis vs + extended }\end{array}$ & $\begin{array}{l}1.987 \\
1.549\end{array}$ & $\begin{array}{l}0.906-4.357 \\
0.769-3.120\end{array}$ & $\begin{array}{l}0.0865 \\
0.2207\end{array}$ & & & \\
\hline
\end{tabular}

Abbreviations: AC, adenocarcinoma; ASC, adenosquamous carcinoma; $\mathrm{Cl}$, confidence interval; HR, hazard ratio; LN, lymph node; LNR, lymph node ratio; MIS, minimally invasive surgery; SCC, squamous cell carcinoma.

contrast, low LNR can result from more aggressive LN dissection, which correlated with better survival outcomes. For this reason, we only included women who had at least 10 total LNs removed according to final pathological reports, and this may help reflect the exact tumor burden using LNR. In this study, the cut-off value of LNR for predicting tumor recurrence and death was $6.25 \%$, according to ROC curve analyses. In univariate analysis, high LNR was associated with worse DFS (HR, 25.577; 95\% CI, 7.015-108.408; $p<$ $0.0001)$ and OS (HR, 10.556; 95\% CI, 1.298-85.844; $p=$ 0.0275). However, after adjusting clinicopathological parameters to LNR, only DFS was associated with LNR (HR, 5.930; 95\% CI, 2.114-16.634; $p=0.0007$ ).
We performed a subgroup analysis according to LN metastasis status. Only LNR could predict tumor recurrence in patients with $\mathrm{LN}$ metastasis; however, no $\mathrm{LN}$ characteristics were associated with prognosis in patients without LN metastasis. The association between the number of removed LN and prognosis according to LN metastasis status was controversial. Kenter et al extensive LN dissection resulted in longer OS and DFS for the positive LN group but not the negative LN group. ${ }^{19}$ Alternatively, Shah et al showed that extensive LN dissection had no survival benefit in positive LN patients; however, it was associated with improved survival in negative $\mathrm{LN}$ patient. ${ }^{20}$ Mao et al demonstrated that the number of 
Table 3 Univariate and Multivariate Analyses of Clinical Variables and Lymph Node Characteristics for Prediction of Death

\begin{tabular}{|c|c|c|c|c|c|c|}
\hline \multirow[t]{2}{*}{ Variables } & \multicolumn{3}{|c|}{ Univariate Analysis } & \multicolumn{3}{|c|}{ Multivariate Analysis } \\
\hline & HR & $95 \% \mathrm{Cl}$ & $\mathbf{P}$ & HR & $95 \% \mathrm{Cl}$ & $P$ \\
\hline $\begin{array}{l}\text { Age (years) } \\
\leq 40 \text { vs }>40\end{array}$ & 2.557 & $0.611-10.694$ & 0.1985 & & & \\
\hline $\begin{array}{l}\text { Stage } \\
\geq \mid \mathrm{IB} 2 \text { vs }|\mathrm{B}|\end{array}$ & 3.895 & $1.154-13.145$ & 0.0284 & 3.349 & $1.168-9.608$ & 0.0246 \\
\hline $\begin{array}{l}\text { Histology } \\
\text { AC/ASC vs SCC }\end{array}$ & 3.068 & $1.002-9.392$ & 0.0495 & 3.041 & $1.050-8.807$ & 0.0404 \\
\hline $\begin{array}{l}\text { Tumor size } \\
>2 \mathrm{~cm} \text { vs } \leq 2 \mathrm{~cm}\end{array}$ & 2.300 & $0.805-6.569$ & 0.1198 & & & \\
\hline Lymphovascular invasion & 1.462 & $0.484-4.410$ & 0.5006 & & & \\
\hline Deep stromal invasion & 1.949 & $0.67 \mid-5.665$ & 0.2201 & & & \\
\hline Parametrial invasion & 2.931 & $0.545-15.749$ & 0.2101 & & & \\
\hline Positive margin & 2.242 & $0.458-10.967$ & 0.3190 & & & \\
\hline $\begin{array}{l}\text { Type of surgery } \\
\text { MIS vs open }\end{array}$ & $\mathrm{I} .175$ & $0.176-7.855$ & 0.8682 & & & \\
\hline $\begin{array}{l}\text { Total } L N \text { counts } \\
\leq 30 \text { vs }>30\end{array}$ & 1.160 & $0.397-3.392$ & 0.7864 & & & \\
\hline LN metastasis & 3.672 & $0.916-14.730$ & 0.0664 & & & \\
\hline $\begin{array}{l}\text { Total positive LN counts } \\
>\text { I vs } \leq \text { I }\end{array}$ & 1.943 & $0.308-12.25 \mid$ & 0.4796 & & & \\
\hline $\begin{array}{l}\text { LNR } \\
>0.0625 \text { vs } \leq 0.0625\end{array}$ & 10.556 & $1.298-85.844$ & 0.0275 & & & \\
\hline $\begin{array}{l}\text { Level of LN dissection } \\
\text { Pelvis vs }+ \text { low paraaortic } \\
\text { Pelvis vs }+ \text { extended }\end{array}$ & $\begin{array}{l}1.935 \\
1.378\end{array}$ & $\begin{array}{l}0.503-7.449 \\
0.424-4.476\end{array}$ & $\begin{array}{l}0.3372 \\
0.5935\end{array}$ & & & \\
\hline
\end{tabular}

Abbreviations: AC, adenocarcinoma; ASC, adenosquamous carcinoma; LN, lymph node; LNR, lymph node ratio; MIS, minimally invasive surgery; SCC, squamous cell carcinoma.

LNs removed was not an independent prognostic factor for patients with LN-negative early-stage cervical cancer. ${ }^{21}$ In our study, the number of LN removal was not associated with prognosis regardless of $\mathrm{LN}$ metastasis status. Primarily, both total LN counts and level of LN dissection was not associated with survival outcomes in the LNnegative group. Sentinel node biopsy may replace the systemic LN dissection, especially in LN-negative earlystage cervical cancer.

The role of systemic lymphadenectomy is to confirm regional nodal metastasis, which is the most crucial factor in determining adjuvant therapy. Moreover, the benefits of lymphadenectomy may extend beyond merely detecting metastatic diseases, that is, the removal of any micrometastases and primary stations of lymph drainage. However, the extent and technique used for nodal dissection are still controversial. A previous study showed that extended, systematic LN dissection based on ontogenetic anatomy resulted in high regional tumor control without adjuvant radiation. ${ }^{22}$ Also, Ungar et al demonstrated that laterally extended parametrectomy provided comparable survival outcomes for stage IB cervical cancer patients with pelvic nodal metastasis without adjuvant radiotherapy. ${ }^{23}$ Previously, we showed that surgical technique and outcomes 

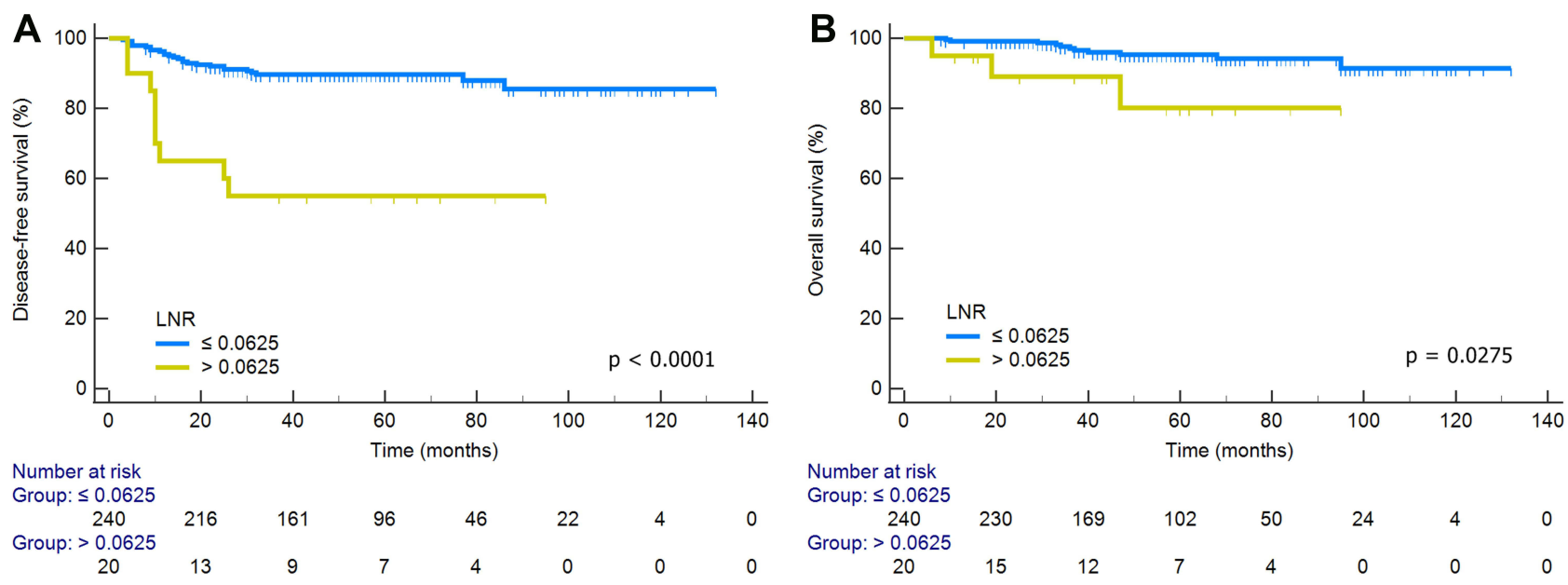

Figure I Kaplan-Meier survival plots of disease-free survival (A) and overall survival (B) according to the lymph node ratio.

of extended lymphadenectomy during radical hysterectomy and expected extended lymphadenectomy might improve survival outcomes. This study, however, extended lymphadenectomy did not improve survival outcomes.

The removal of pelvic LNs has shown poor clinical effects, including nerve damage, lymphedema, and lymphocyst formation. ${ }^{24}$ Sentinel LN mapping may be considered as a contemporary technique that could provide additional benefits over traditional pelvic lymphadenectomy, especially in early-stage cervical cancer. ${ }^{25}$ In this study, LNR $(>0.0625)$ and the number of positive nodes $(>1)$ were associated with tumor recurrence. Therefore, patients with $<1$ positive node in preoperative imaging studies may be considered for sentinel LN mapping during radical hysterectomy.

This study has several limitations; first, selection bias might exist due to retrospective design. Second, our results may not be generalized owing to the relatively small sample size in a single institution. Our study offers some unique and significant findings despite these limitations, and it differs from previous studies. We evaluated the prognostic value of various $\mathrm{LN}$ characteristics, including LNR, and conducted a subgroup analysis of LN characteristics according to LN metastasis status. Furthermore, survival benefits were evaluated according to the level of lymphadenectomy.

In conclusion, LNR was the most robust biomarker to predict tumor recurrence among various LN characteristics. Total retrieved LN counts and level of lymphadenectomy did not affect survival outcomes. Our results may provide valuable prognostic information to physicians and make it possible to personalize treatment that may involve more aggressive adjuvant therapy.

Table 4 Multivariate Analyses of Lymph Node Characteristics in Relation to Tumor Recurrence and Death After Adjusting for Clinicopathologic Factors

\begin{tabular}{|c|c|c|c|c|c|c|}
\hline \multirow[t]{2}{*}{ Variables } & \multicolumn{3}{|c|}{ Disease-Free Survival } & \multicolumn{3}{|c|}{ Overall Survival } \\
\hline & HR & $95 \% \mathrm{Cl}$ & $P$ value & HR & $95 \%$ CT & $P$ value \\
\hline Total LN counts & I.44I & $0.677-3.069$ & 0.0763 & 0.886 & $0.282-2.776$ & 0.8348 \\
\hline LN metastasis & 1.664 & $0.679-4.074$ & 0.2655 & 1.832 & $0.431-7.778$ & 0.7623 \\
\hline Total positive LN counts & 3.632 & $1.107-11.922$ & 0.0334 & 0.698 & $0.113-4.309$ & 0.6987 \\
\hline LNR & 5.930 & $2.114-16.634$ & 0.0007 & 2.003 & $0.418-9.585$ & 0.3845 \\
\hline Low paraaortic LN dissection & 1.415 & $0.584-3.430$ & 0.4418 & 1.246 & $0.279-5.568$ & 0.7736 \\
\hline Extended LN dissection & 1.098 & $0.52 I-2.314$ & 0.8069 & 1.039 & $0.303-3.566$ & 0.9519 \\
\hline
\end{tabular}

Abbreviations: $\mathrm{Cl}$, confidence interval; HR, hazard ratio; LN, lymph node; LNR, lymph node ratio; SCC, squamous cell carcinoma. 


\section{Disclosure}

The authors report no potential conflicts of interest relevant to this article.

\section{References}

1. Peters WA 3rd, Liu PY, Barrett RJ 2nd, et al. Concurrent chemotherapy and pelvic radiation therapy compared with pelvic radiation therapy alone as adjuvant therapy after radical surgery in high-risk early-stage cancer of the cervix. $J$ Clin Oncol. 2000;18 (8):1606-1613. doi:10.1200/JCO.2000.18.8.1606

2. Sedlis A, Bundy BN, Rotman MZ, Lentz SS, Muderspach LI, Zaino RJ. A randomized trial of pelvic radiation therapy versus no further therapy in selected patients with stage IB carcinoma of the cervix after radical hysterectomy and pelvic lymphadenectomy: a gynecologic oncology group study. Gynecol Oncol. 1999;73 (2):177-183. doi:10.1006/gyno.1999.5387

3. Delgado G, Bundy B, Zaino R, Sevin BU, Creasman WT, Major F. Prospective surgical-pathological study of disease-free interval in patients with stage IB squamous cell carcinoma of the cervix: a gynecologic oncology group study. Gynecol Oncol. 1990;38 (3):352-357. doi:10.1016/0090-8258(90)90072-s

4. Huang BX, Fang F. Progress in the study of lymph node metastasis in early-stage cervical cancer. Curr Med Sci. 2018;38(4):567-574. doi:10.1007/s11596-018-1915-0

5. Pieterse QD, Kenter GG, Gaarenstroom KN, et al. The number of pelvic lymph nodes in the quality control and prognosis of radical hysterectomy for the treatment of cervical cancer. Eur J Surg Oncol. 2007;33(2):216-221. doi:10.1016/j.ejso.2006.09.037

6. Ditto A, Martinelli F, Lo Vullo S, et al. The role of lymphadenectomy in cervical cancer patients: the significance of the number and the status of lymph nodes removed in 526 cases treated in a single institution. Ann Surg Oncol. 2013;20(12):3948-3954. doi:10.1245/ s10434-013-3067-6

7. Lu H, Guo R, Yang H, et al. The prognostic value of negative lymph node count for patients with cervical cancer after radical surgery. Oncotarget. 2017;9(2):2810-2818. doi:10.18632/oncotarget.23596

8. Aoki Y, Sasaki M, Watanabe $M$, et al. High-risk group in node-positive patients with stage IB, IIA, and IIB cervical carcinoma after radical hysterectomy and postoperative pelvic irradiation. Gynecol Oncol. 2000;77(2):305-309. doi:10.1006/gyno.2000.5788

9. Joo JH, Kim YS, Nam JH. Prognostic significance of lymph node ratio in node-positive cervical cancer patients. Medicine. 2018;97 (30):e11711. doi:10.1097/MD.0000000000011711

10. Aslan K, Meydanli MM, Oz M, Tohma YA, Haberal A, Ayhan A. The prognostic value of lymph node ratio in stage IIIC cervical cancer patients triaged to primary treatment by radical hysterectomy with systematic pelvic and para-aortic lymphadenectomy. J Gynecol Oncol. 2020;31(1):e1. doi:10.3802/jgo.2020.31.e1

11. Kim SI, Kim TH, Lee M, et al. Lymph node ratio is a strong prognostic factor in patients with early-stage cervical cancer undergoing minimally invasive radical hysterectomy. Yonsei Med J. 2021;62 (3):231-239. doi:10.3349/ymj.2021.62.3.231
12. Kehoe SM, Miller DS. The role of lymphadenectomy in endometrial cancer. Curr Treat Options Oncol. 2006;7(2):93-101. doi:10.1007/ s11864-006-0044-3

13. Lim S, Cho K, Lee S, et al. Effect of number of retrieved lymph nodes on prognosis in FIGO stage IB-IIA cervical cancer patients treated with primary radical surgery. J Obstet Gynaecol Res. 2017;43 (1):211-219. doi:10.1111/jog.13171

14. Lee YS, Chong GO, Lee YH, Hong DG, Cho YL, Park IS. Robotassisted total preservation of the pelvic autonomic nerve with extended systematic lymphadenectomy as part of nerve-sparing radical hysterectomy for cervical cancer. Int J Gynecol Cancer. 2013;23 (6):1133-1138. doi:10.1097/IGC.0b013e31829b10db

15. Ramirez PT, Frumovitz M, Pareja R, et al. Minimally invasive versus abdominal radical hysterectomy for cervical cancer. $N$ Engl J Med. 2018;379(20):1895-1904. doi:10.1056/NEJMoa1806395

16. Pecorelli S. Revised FIGO staging for carcinoma of the vulva, cervix, and endometrium. Int J Gynaecol Obstet. 2009;105(2):103-104. doi:10.1016/j.ijgo.2009.02.012

17. Querleu D, Morrow CP. Classification of radical hysterectomy. Lancet Oncol. 2008;9(3):297-303. doi:10.1016/S1470-2045(08) 70074-3

18. Kwon J, Eom KY, Kim YS, et al. The prognostic impact of the number of metastatic lymph nodes and a new prognostic scoring system for recurrence in early-stage cervical cancer with high risk factors: a multicenter cohort study (KROG 15-04). Cancer Res Treat. 2018;50(3):964-974. doi:10.4143/crt.2017.346

19. Kenter GG, Hellebrekers BW, Zwinderman KH, van de Vijver M, Peters LA, Trimbos JB. The case for completing the lymphadenectomy when positive lymph nodes are found during radical hysterectomy for cervical carcinoma. Acta Obstet Gynecol Scand. 2000;79 (1):72-76. doi:10.1034/j.1600-0412.2000.079001072.x

20. Shah M, Lewin SN, Deutsch I, et al. Therapeutic role of lymphadenectomy for cervical cancer. Cancer. 2011;117(2):310-317. doi:10.1002/cncr.25408

21. Mao S, Dong J, Li S, Wang Y, Wu P. Prognostic significance of number of nodes removed in patients with node-negative early cervical cancer. J Obstet Gynaecol Res. 2016;42(10):1317-1325. doi:10.1111/jog. 13058

22. Höckel M, Horn LC, Fritsch H. Association between the mesenchymal compartment of uterovaginal organogenesis and local tumour spread in stage IB-IIB cervical carcinoma: a prospective study. Lancet Oncol. 2005;6(10):751-756. doi:10.1016/S1470-2045(05) 70324-7

23. Ungár L, Pálfalvi L, Tarnai L, Horányi D, Novák Z. Surgical treatment of lymph node metastases in stage IB cervical cancer. The laterally extended parametrectomy (LEP) procedure: experience with a 5 year follow-up. Gynecol Oncol. 2011;123(2):337-341. doi:10.1016/j.ygyno.2011.07.001

24. Matsuura Y, Kawagoe T, Toki N, Tanaka M, Kashimura M. Longstanding complications after treatment for cancer of the uterine cervix-clinical significance of medical examination at 5 years after treatment. Int J Gynecol Cancer. 2006;16(1):294-297. doi:10.1111/ j.1525-1438.2006.00354.x

25. Wang L, Liu S, Xu T, Yuan L, Yang X. Sentinel lymph node mapping in early-stage cervical cancer: meta-analysis. Medicine. 2021;100 (34):e27035. doi:10.1097/MD.0000000000027035 


\section{Publish your work in this journal}

Cancer Management and Research is an international, peer-reviewed open access journal focusing on cancer research and the optimal use of preventative and integrated treatment interventions to achieve improved outcomes, enhanced survival and quality of life for the cancer patient.
The manuscript management system is completely online and includes a very quick and fair peer-review system, which is all easy to use. Visit http://www.dovepress.com/testimonials.php to read real quotes from published authors. 